\title{
Diseño de un sistema de análisis temporal y espectral para detectar fallas por vibración en motores eléctricos
}

\author{
A Temporal and Spectral Analussis Sustem Design, to Detect \\ Vibration Failures in Electrical Motors
}

\section{Desenho de um sistema de análise temporal e espectral para detectar falhas por vibração em motores elétricos}

Fecha de Recepción: 3 de Septiembre de 2014

Fecha de Aceptación: 9 de Noviembre de 2014
Francisco Ernesto Moreno-García* José Armando Becerra-Vargas** Carlos Andrés Rendón-Echeverri***

\section{Resumen}

El documento se basa en el desarrollo experimental de un sistema de medición y análisis de vibraciones para brindar un estudio de las más relevantes variables utilizadas en el análisis espectral de motores eléctricos, con el fin de detectar posibles fallas. Dicho sistema de análisis fue implementado en un banco de pruebas elaborado por el Grupo de Investigación en Automatización y Control (GIAC), de la Universidad Francisco de Paula Santander.

Palabras clave: Aceleración, Análisis de vibraciones, Análisis espectral, Desplazamiento, Motor eléctrico,

\section{Abstract}

This document is based on the experimental development of a measurement and analysis vibrations system; to provide a study of the most relevant variables used in the spectral analysis of electric motors, in order to detect possible faults. This analysis system was implemented in a test bank developed by The Research Group in Automation and Control (GIAC) of the University Francisco de Paula Santander.

Keywords: Acceleration, Displacement, Electric Motor, Spectrum Analysis, Vibration.

* PhD. Universidad Francisco de Paula Santander (San José de Cúcuta-Norte de Santander, Colombia). femgarcia@ufps.edu.co

** M. Sc. Universidad Francisco de Paula Santander (San José de Cúcuta-Norte de Santander, Colombia). josearmandobv@ufps.edu.co

*** Ing. Electromecánico. Universidad Francisco de Paula Santander (San José de Cúcuta-Norte de Santander, Colombia). carlosrendon02@gmail.com 


\section{Resumo}

O documento se baseia no desenvolvimento experimental de um sistema de medição e análise de vibrações para fornecer um estudo das mais relevantes variáveis utilizadas na análise espectral de motores elétricos, com o objetivo de detectar possíveis falhas. Tal sistema de análise foi implementado em um banco de provas elaborado pelo "Grupo de Investigación en Automatización y Control" (GIAC), da Universidade Francisco de Paula Santander.

Palavras chave: Aceleração, Análise de vibrações, Análise espectral, Deslocamento, Motor elétrico. 


\section{INTRODUCCIÓN}

El objetivo principal de analizar y diagnosticar el estado de una máquina es determinar las medidas necesarias para corregir la condición de vibración y reducir así el nivel de las fuerzas vibratorias indeseadas e innecesarias; en consecuencia, al estudiar los datos, el interés principal deberá ser la identificación de las amplitudes predominantes de la vibración, la determinación de sus causas y la corrección del problema que ellas generan [1].

El mantenimiento de equipos e instalaciones industriales ha cobrado gran importancia en las últimas décadas, debido a que las exigencias de calidad en la producción son cada vez mayores y al hecho de que las empresas necesitan ser más competitivas en el ámbito del comercio internacional [2].

La aplicación de técnicas avanzadas para el mantenimiento requiere tener un conocimiento preciso de las máquinas, de sus partes componentes y de su historial de funcionamiento; esto también incluye el estudio vibro-dinámico, que permita monitorear $\mathrm{y}$ supervisar las variables con sus valores de frontera (alarma) para condiciones reales de exploración de los motores. La adaptación a las condiciones reales de montaje y explotación de cada máquina es un elemento importante para obtener los resultados principales de la aplicación del mantenimiento predictivo en motores eléctricos y otras máquinas. La obtención de firmas espectrales personalizadas facilita considerablemente el seguimiento de las variables de pronóstico [3].

Tradicionalmente, las máquinas eléctricas se consideraban elementos con bajo índice de averías, sobre todo si se trataba de motores Jaula de ardilla; sin embargo, la tendencia actual de diseñar máquinas de bajo costo hace que los materiales utilizados trabajen más cerca de sus límites de resistencia mecánica y electromagnética; por este motivo, y en especial cuando el motor trabaja en ambientes hostiles, la posibilidad de deterioro progresivo se ha incrementado [3].

Teniendo en cuenta que con frecuencia los motores eléctricos se encuentran en puntos vitales de gran complejidad, y que los criterios industriales actuales tienden hacia una producción de elevada calidad y sin interrupciones, se hace cada vez más necesario un control sobre su funcionamiento para determinar los indicios de una posible avería [4].

\section{VIBRACIONES EN MOTORES ELÉCTRICOS}

La vibración es el movimiento armónico de una máquina, o elemento de ella, en cualquier dirección del espacio desde su posición de equilibrio. Generalmente, la causa de la vibración reside en problemas mecánicos, como son: desequilibrio de elementos rotativos, desalineación en acoplamientos, engranajes desgastados o dañados y rodamientos deteriorados; en fuerzas aerodinámicas o hidráulicas, o en problemas eléctricos. Estas causas se detectan estudiando las características de vibración.

Las características más importantes de las vibraciones son: frecuencia, desplazamiento, velocidad, aceleración y energía de impulsos. La frecuencia es una característica simple y significativa en este análisis; se define como el número de ciclos completos en un período de tiempo, y su unidad característica es cpm (ciclos por minuto). Los diferentes problemas son detectados por las frecuencias iguales a la velocidad de giro, o bien múltiplos suyos.

La amplitud de la vibración indica la importancia, o gravedad del problema; esta característica da una idea de la condición de la máquina. Se podrá medir la amplitud de desplazamiento, la velocidad o la aceleración. La energía de impulsos proporciona información importante a la hora de analizar vibraciones; este parámetro mide los impulsos de energía de vibración de breve duración y, por lo tanto, de alta frecuencia; con esta medida se encuentran rápidamente las vibraciones a altas frecuencias provocadas por estos defectos. El valor de la energía de impulsos es básicamente una medida de aceleración, pero tiene como unidad g-SE [5].

Las etapas para medir y, posteriormente, analizar una vibración, que constituyen la cadena de medición, son:

- Etapa transductor

- Etapa de acondicionamiento de la señal

- Etapa de análisis o medición

- Etapa de registro 
Los transductores típicamente utilizados para la medición de vibraciones debido a sus características son los sensores acelerómetros. Un acelerómetro es un instrumento para medir la aceleración de un objeto al que va unido; son sensores inerciales que miden la segunda derivada de la posición $[6,7]$.

La adquisición de datos es el primer y principal paso para hacer un análisis de vibraciones. Los datos (desplazamiento, velocidad o aceleración) dependerán de la velocidad de la máquina, de acuerdo con su relación equivalente de frecuencia $(\mathrm{rpm}=\mathrm{cpm})$. Así, para bajas rpm (bajos cpm) se tomarán datos de desplazamientos; para velocidades que estén dentro del orden de los 600 y 60.000 rpm se medirán velocidades, y para las que sean de orden superior, los datos a tomar serán aceleraciones. En la Fig. 1 se muestran los sentidos principales de medición a partir de la utilización de un sensor de tipo acelerómetro como elemento de medición, en donde la vibración se tomará generalmente en rodamientos de la máquina o puntos donde sea más probable un fallo por acoplamiento o equilibrio, puntos donde se transmitan las fuerzas vibratorias a través de un acelerómetro.

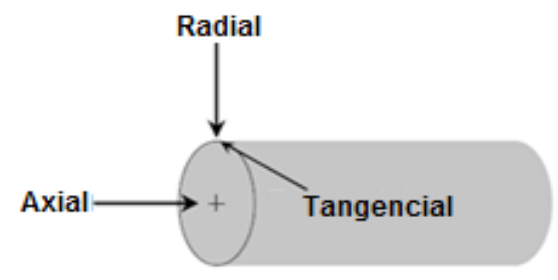

Fig. 1. Sentidos principales de la medición con un acelerómetro [4]

\section{ANÁLISIS DE VIBRACIONES EN MOTORES ELÉCTRICOS}

El objetivo del análisis de vibraciones es extraer de ellas el máximo de información relevante; para esto existen diferentes técnicas de análisis, tanto en el dominio tiempo como en el dominio frecuencia, las cuales tienen sus propias ventajas para algunas aplicaciones en particular.
Para desarrollar el sistema planteado fue necesario definir claramente su estructura mediante la identificación de las diferentes etapas del proceso, para lo cual se establece el diagrama de bloque mostrado en la Fig. 2, que plantea el sistema para el análisis de fallas ocasionadas por vibraciones en motores eléctricos.

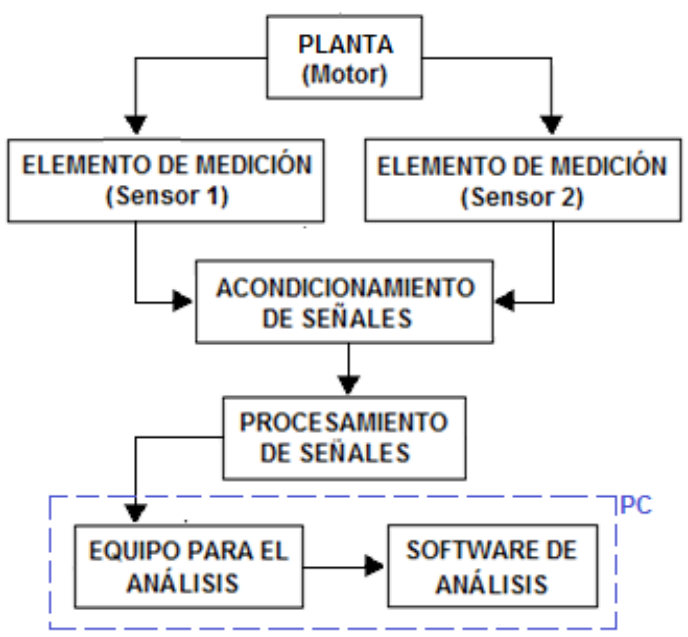

Fig. 2. Diagrama de bloques del sistema para el análisis de fallas por vibraciones en motores eléctricos

Del diagrama de bloques del sistema para el análisis de fallas de la Fig. 2 se puede deducir que para el sistema planteado se implementaron dos sensores y que el equipo de análisis es un PC que contiene el software por medio del cual se diseñará el sistema.

\section{BANCO DE PRUEBAS EXPERIMENTAL}

\section{A. Planta: motor-freno electromagnético}

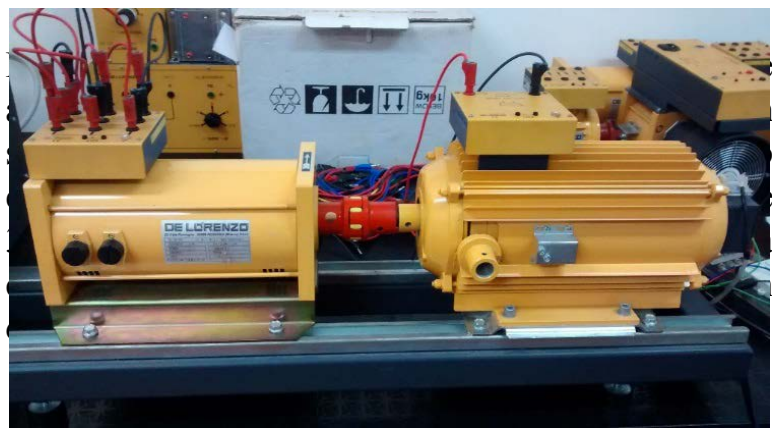

Fig. 3. Planta para toma de medidas de aceleración para la detección de fallas por vibración 


\section{B. Elementos de medición: acelerómetro}

\section{MMA7361}

Los elementos de medición utilizados se constituyen por una placa basada en el sensor MMA7361L, que es un acelerómetro de tres ejes con salidas de tensión analógicas, con un rango de sensibilidad ajustable $\mathrm{a} \pm 1.5 \mathrm{~g}$ o $\pm 6 \mathrm{~g}$, la cual es medida con respecto a la constante gravedad. Los pines que se van a utilizar para la implementación de los acelerómetros son los correspondientes al voltaje de alimentación de los sensores (Vcc y Gnd), las salidas, el pin de $g$-Select, el pin Sleep y el de 3.3V, cuya conexión se realiza de la manera como se indica en la Fig. 4.

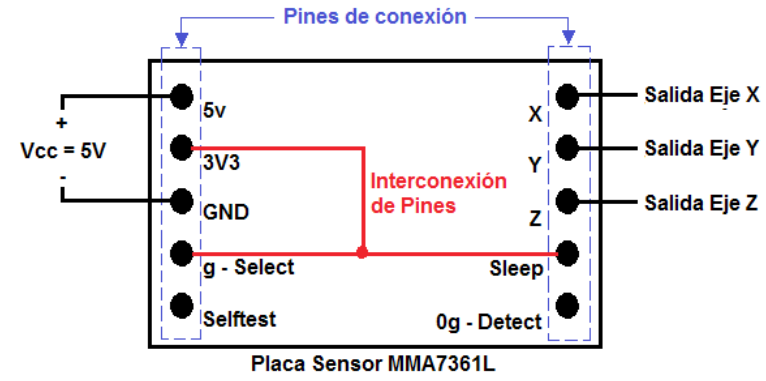

Fig. 4. Conexión de los sensores MMA7361L para el proceso de medición de aceleración

Según la hoja de especificaciones técnicas de los sensores, el voltaje de alimentación típico del sensor es de $5 \mathrm{~V}_{\mathrm{DC}}$. Cuando es alimentado el sensor con el voltaje típico mencionado anteriormente, el pin de $3.3 \mathrm{~V}$ actúa como una salida, que es utilizada como estado digital (1 ó 0) para la selección del rango de sensibilidad, que se establece en $\pm 6 \mathrm{~g}$, para lo cual se realiza la conexión del pin $g$-Select (selector de rango) al pin de $3.3 \mathrm{~V}$; de igual modo, al verificar el encendido se debe probar que está generando respuesta en las salidas, de lo contrario, como se presentó en este caso, la ausencia de respuesta inicial indica que el sensor se encuentra inactivo, para lo cual es necesario poner en estado 1 el pin Sleep, es decir, realizar la conexión entre el pin de $3.3 \mathrm{~V}$ y el pin Sleep.

Con el propósito de permitir la utilización de los sensores para la medición en diversos puntos del motor, evitando disponer de placas para la fijación definitiva del sensor al motor, se construyó una estructura para el sensor, en la cual se utiliza un imán para la fijación de dicho sensor en cualquier punto de la máquina; este imán es adherido a una placa de acrílico utilizada para aislar la placa del sensor de superficies que puedan ocasionarle daños, además de proporcionar aislamiento. Finalmente, la estructura implementada con los sensores para llevar a cabo la medición de vibraciones en diferentes puntos del motor eléctrico queda como se muestra en la Fig. 5.
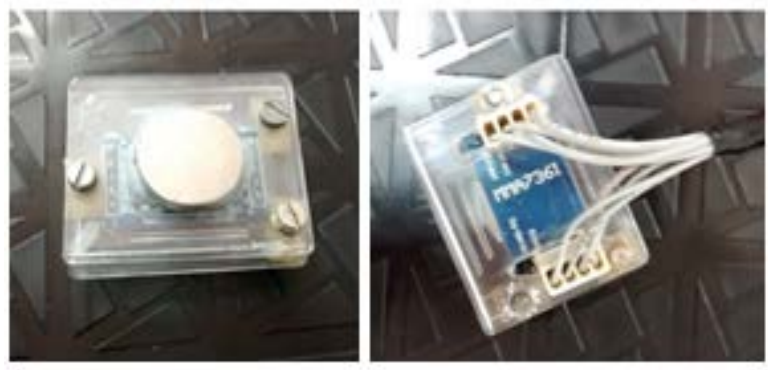

Fig. 5. Estructura de los sensores para medir vibraciones en diferentes puntos del motor eléctrico

\section{Procesamiento de señales: DAQ NI USB- 6009}

Las salidas de los ejes del sensor utilizado se encuentran determinadas en un rango de sensibilidad de $\pm 6 \mathrm{~g}$, con lo cual, según la hoja de especificaciones técnicas, tienen una resolución típica de $206 \mathrm{mV} / \mathrm{g}$; esto quiere decir que para una medición máxima de aceleración de $\pm 6 \mathrm{~g}$ en cualquier eje del sensor, se obtendría un voltaje de salida de $\pm 1.236 \mathrm{~V}$. Este valor de voltaje de salida, normalmente, se acondicionaría a estándares de $0 \mathrm{~V}$ a $10 \mathrm{~V}$ o de $1 \mathrm{~V}$ a $10 \mathrm{~V}$, pero esta etapa no se implementó, ya que se utiliza como elemento de recepción y transmisión de datos una tarjeta DAQ NI USB-6009 que tiene entradas analógicas con un rango de $\pm 10 \mathrm{~V}$, es decir, permite la entrada de voltajes negativos hasta de $-10 \mathrm{~V}$, con lo cual se cubre ampliamente la salida de voltaje negativo de los sensores. La configuración realizada para las mediciones de entrada y sus características en la tarjeta de adquisición de datos DAQ NI USB-6009 se muestra en la Fig. 6. 


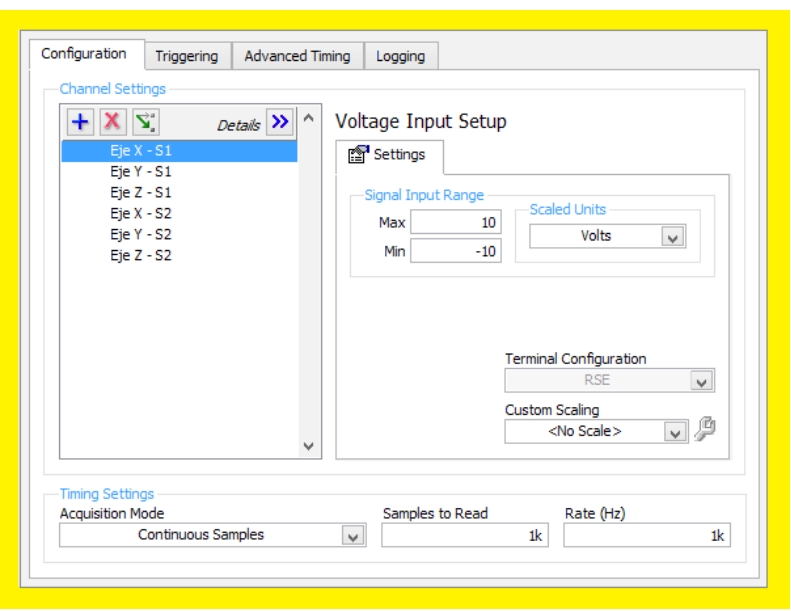

Fig. 6. Configuración de las entradas de medidas y sus características mediante el bloque DAQ Assistant en LabVIEW

\section{Programación del sistema de ANÁLISIS}

La elaboración del sistema de análisis para detección de fallas por vibraciones en motores eléctricos se realiza en el software LabVIEW ${ }^{\circledR}$ de National Instruments. A continuación se presenta la descripción de las etapas relevantes del proceso de elaboración del diagrama de bloques de programación implementado para el sistema de análisis de las señales de aceleración medidas en el motor eléctrico.

\section{A. Importación de señales}

La importación de las señales de aceleración medidas en el motor eléctrico a la ventana de programación de LabVIEW ${ }^{\circledR}$ se desarrolló usando el bloque DAQ Assitanty el bloque de Split Signals, como se muestra en la Fig. 7, cuya función es dividir una señal de entrada combinada en dos o más señales de componente, en donde cada una de éstas contiene la información de tiempo de la forma de onda. De esta manera se obtuvieron de manera separada los datos de la medición realizada en cada eje por los dos sensores implementados.

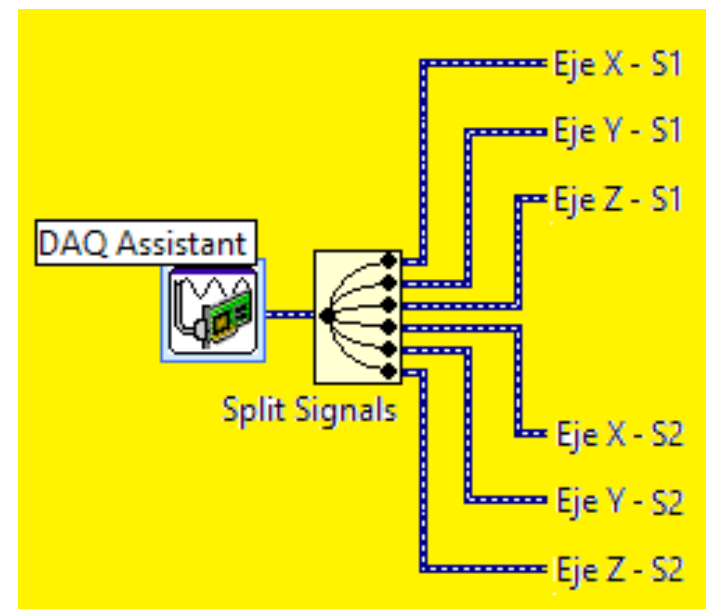

Fig. 7. Diagrama de bloques para la importación de datos de medición en LabVIEW

\section{B. Análisis temporal}

En el análisis temporal se establecieron los datos y gráficas de cada una de las señales de aceleración medidas en cada uno de los ejes de los sensores en el dominio del tiempo. A continuación se describe el proceso realizado para el análisis de los datos en el tiempo, teniendo en cuenta de forma general para las seis señales de datos que representan los ejes $\mathrm{X}-\mathrm{Y}-\mathrm{Z}$ de los dos sensores utilizados.

1) Ajuste de datos de la señal de entrada: En primer lugar se realizó un ajuste de los datos de la señal de entrada, para lo cual se tiene en cuenta la resolución de la medida, el ajuste de offset y la unidad de la medición de la aceleración.

Para obtener datos exactos de la señal medida con respecto a la unidad de aceleración, se ajustó la resolución de los datos por medio de un bloque matemático de división, en donde la señal de entrada de la medición es dividida por el valor de la resolución, quedando la medida de aceleración de dicha señal referenciada con respecto al rango de fuerza $g$.

El ajuste de offset depende de la posición en la que se ubique el sensor para la medición de la variable de aceleración, por lo tanto, se compensó a través de un bloque matemático de resta para sustraer el valor del offset de la señal de entrada, el cual se establece como un control numérico, para así poder cambiar desde la interfaz HMI el valor de offset si es necesario. Adicionalmente, se obtuvieron los datos de la señal 
de aceleración medida con respecto a la unidad del sistema internacional de unidades en $\mathrm{m} / \mathrm{s}^{2}$ a través de un bloque matemático de multiplicación que relaciona el valor de la constante de gravedad.

La implementación de los bloques anteriormente descritos para el ajuste de datos de la señal de entrada se muestra en la Fig. 8, teniendo en cuenta que se utilizan indicadores gráficos para poder observar el comportamiento de los datos obtenidos en cada salida.

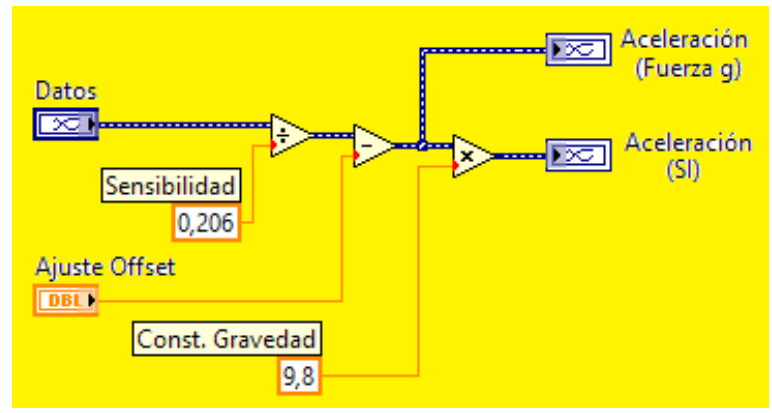

Fig. 8. Diagrama de bloques para el ajuste de datos de la señal de entrada en LabVIEW

2) Obtención de la señal de velocidad: Tomando como referencia los datos de aceleración medidos con respecto al sistema internacional de unidades $\left[\mathrm{m} / \mathrm{s}^{2}\right]$, se pudo obtener la componente de velocidad de la medida a partir de la integración de los datos correspondientes a la aceleración. Para integrar dicha señal se utiliza el bloque de Vibration Level de la librería del Toolkit Sound and Vibration, cuya configuración se realiza como se indica en la Fig. 9.

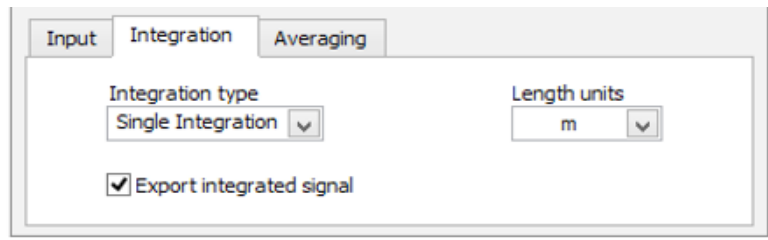

Fig. 9. Configuración del bloque VibrationLevel de LabVIEW para la integración de datos

En la Fig. 10 se puede observar la conexión de los bloques para obtener los datos de aceleración, con su respectivo Convert from Dynamic Data para el tratamiento de datos dinámicos a un arreglo de una dimensión Array of Waveform.

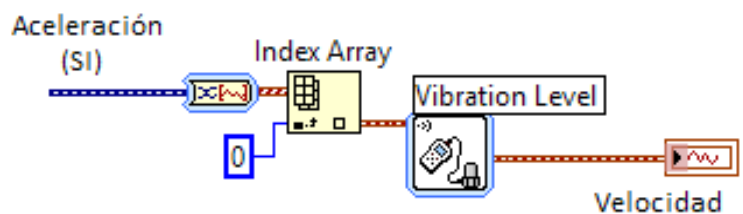

(SI)

Fig. 10. Diagrama de bloques para la obtención de la señal de velocidad en LabVIEW

3) Obtención de la señal de desplazamiento: Tomando como referencia los datos de aceleración medidos con respecto al sistema internacional de unidades $\left(\mathrm{m} / \mathrm{s}^{2}\right)$, también se puede obtener la componente de desplazamiento de la medida a partir de la integración de los datos correspondientes a la aceleración; dicho procedimiento fue apoyado a través de la herramienta Integration Type.

\section{Análisis espectral}

El análisis espectral se realizó con los datos obtenidos de la señal de velocidad para cada uno de los ejes de los dos sensores implementados, a partir del diagrama de bloques mostrado en la Fig. 11.

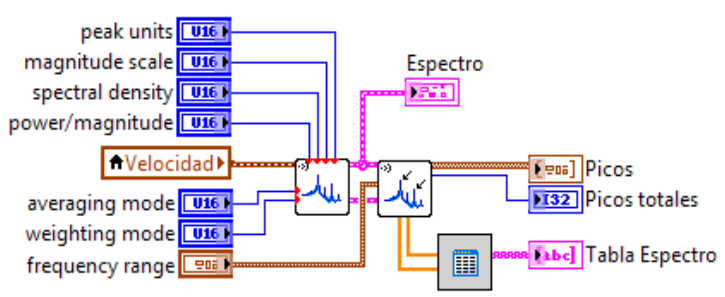

Fig. 11. Diagrama de bloques para realizar el análisis espectral de los datos obtenidos

Para este análisis se trabajó con la señal de datos de velocidad en el dominio del tiempo y en el dominio de la frecuencia. Para el tratamiento de las señales medidas en el dominio de la frecuencia se aplicó el bloque Power Spectrum del Toolkit Sound and Vibration. En la Fig. 11 se presenta la programación Peak Search, cuya función es detectar e indicar los picos altos, junto con la frecuencia en que acontecen, además de obtener información específica a nivel de vibraciones. 


\section{INTERFAZ HOMBRE-MÁQUINA}

Además de realizar una programación basada en diagrama de bloques o interconexión de bloques, a través de LabVIEW se desarrolló paralelamente un panel frontal que representa la interfaz de usuario del código subVI elaborado para el sistema desarrollado en el análisis de vibraciones. En la Fig. 12 se observa la interfaz de usuario en vista completa.

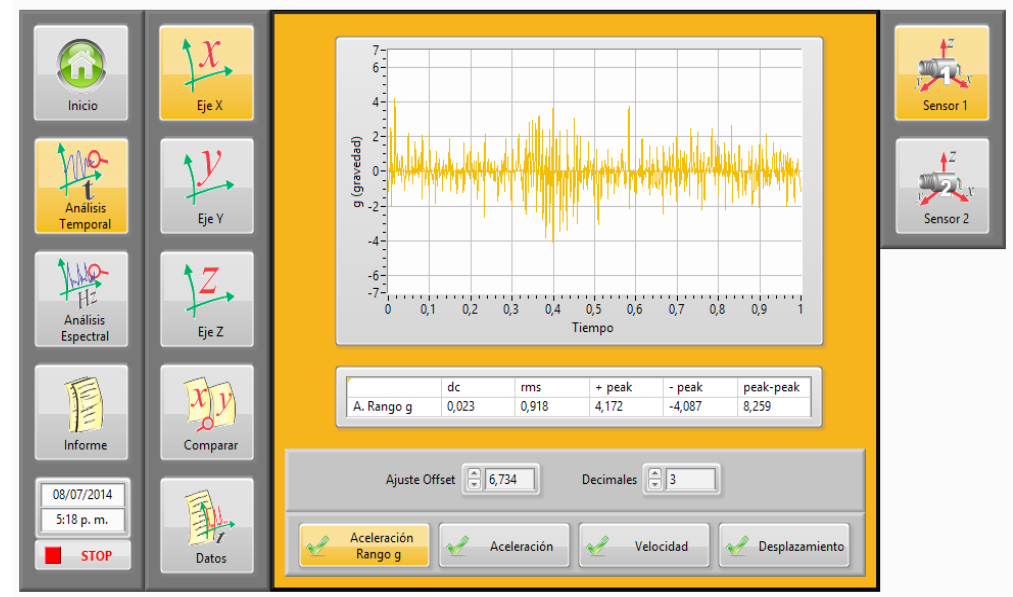

Fig. 12. Interfaz de usuario del sistema de análisis temporal y espectral para la detección de fallas por vibración

La interfaz tiene un menú principal con las opciones de: inicio (presenta la información del sistema), análisis temporal, análisis espectral e informe.

\section{Pruebas experimentales de FUNCIONAMIENTO}

La disposición del banco para la prueba de funcionamiento se muestra en la Fig. 13; dicho banco está conformado por una fuente de alimentación y un motor, que es conectado en delta o triángulo $(\Delta)$ con una alimentación de $220 \mathrm{~V}$, y cuyo eje va unido a un freno electromagnético que hace las veces de carga; de igual manera, el freno electromagnético es conectado a una alimentación de $220 \mathrm{~V}$, pero sólo es activado cuando se desea ejercer sobre el motor eléctrico una carga mayor a la que ejerce el arrastre del eje acoplado; el elemento de medición envía los datos a la tarjeta de adquisición, que posteriormente los transmite al equipo PC.

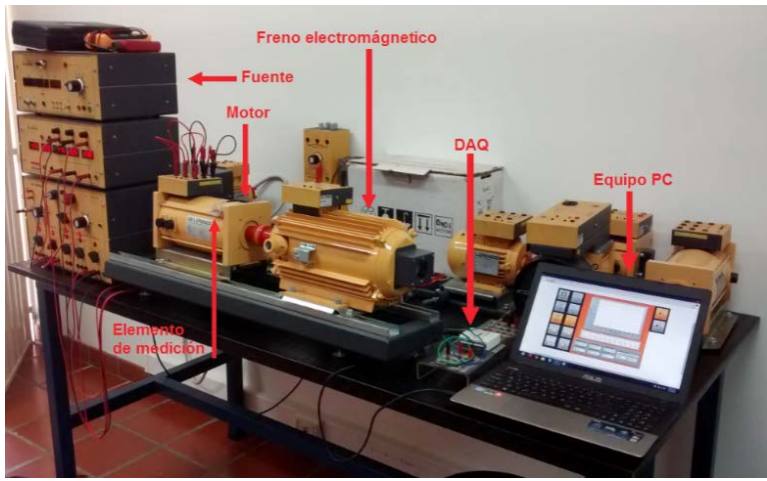

Fig. 13. Equipo experimental para las pruebas de funcionamiento del sistema

Anteriormente se destacó que los sensores se adecuaron en una estructura que permite adherirlos a cualquier superficie del motor eléctrico, con la finalidad de realizar medidas en diferentes puntos de la máquina, dependiendo de las necesidades del análisis. Una vez ubicado el sensor, es fundamental determinar cuál va a ser el sentido de la medición realizada por el. La posición del elemento de medición se determina con base en las indicaciones de la hoja de especificadores del sensor o en las mismas indicaciones en la placa de éste; de esta manera, se establece que los sentidos de la medida (radial, axial y tangencial) corresponden a cada uno de los ejes, de la manera como se indica en la Fig. 14. 


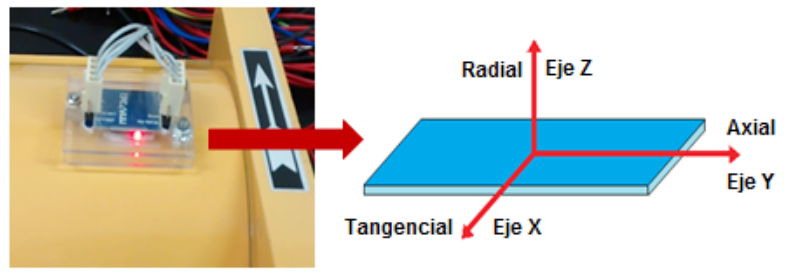

Fig. 14. Sentidos de medición del sensor según su posición

Una vez ubicado el elemento de medición e identificado el sentido de medición realizado por él, se puede proceder con su encendido; posteriormente se inicia el sistema de análisis en LabVIEW, previa verificación de que esté recibiendo señales; si es así, se ajusta el nivel de offset de la medición en cada uno de los ejes, ya que la gravedad de la tierra ejerce una fuerza sobre el elemento de medición dependiendo de la ubicación en la que se encuentre.

El sistema diseñado permite realizar el análisis temporal de la información obtenida a partir de los elementos de medición; no obstante, por motivos prácticos se realiza sólo el análisis espectral de esta información.

En una primera toma de datos, se realizó el análisis para el motor cuando se asume que se encuentra en condiciones ideales de alineamiento; principalmente, se establece un análisis espectral para las medidas tomadas por el sensor 1 , en las cuales el comportamiento fue idéntico en todos sus ejes, por lo cual se toman como referencia los datos obtenidos para el eje X; el espectro de vibración obtenido se muestra en la Fig. 15.

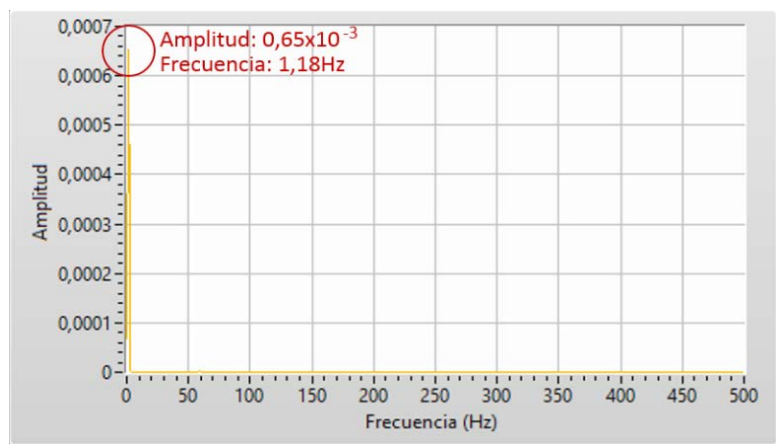

Fig. 15. Espectro de vibración de la primera toma de datos en la prueba de funcionamiento
En el espectro obtenido para el eje X se observa la presencia de un pico con una amplitud de $0.65 \mathrm{~m}$ a una frecuencia de $1.18 \mathrm{~Hz}$, lo que representa, generalmente, la presencia de holguras por tornillos sueltos, lo cual indica además que la máquina se encuentra en buenas condiciones de operación.

Posteriormente, se realizó la segunda toma de datos, en la cual se desalineó el eje del motor con respecto a la carga. Debido a la constitución de la base donde se montó el motor y el freno electromagnético, solo resultaba posible inducir un deslizamiento en dirección radial. Se realizó un análisis espectral para las medidas tomadas por el sensor 1 en el eje Z; esto debido a que en los otros ejes el espectro identificaba picos idénticos pero mucho menores que los presentados en este eje; en la Fig. 16 se observa el espectro de vibración obtenido.

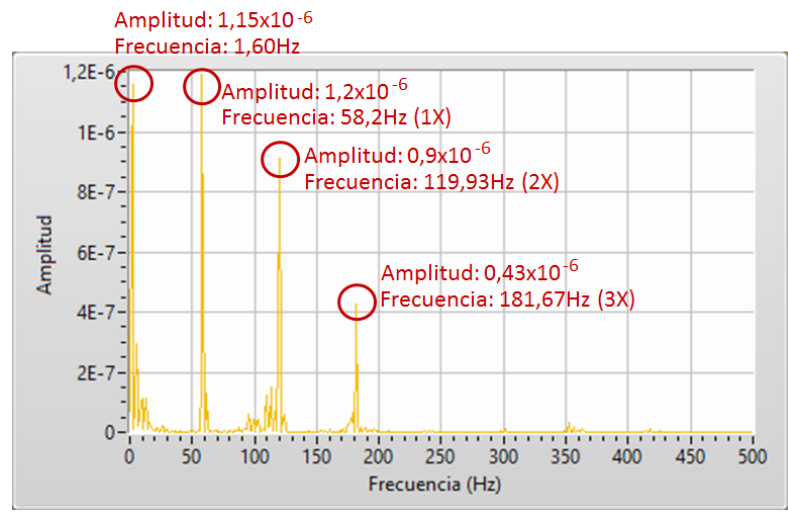

Fig. 16. Espectro de vibración de la segunda toma de datos en la prueba de funcionamiento

En el espectro de la Fig. 16 se observa la presencia de un pico con una amplitud de $1.15 \mu$ a una frecuencia de $1.6 \mathrm{~Hz}$, que de igual manera que en la prueba anterior es atribuido a vibraciones por holguras debidas a tornillos sueltos. De igual manera, se identifican picos con amplitudes de $1.2 \mu, 0.9 \mu$ y $0.43 \mu$ a unas frecuencias de $58.2 \mathrm{~Hz}(1 \mathrm{X}$-frecuencia de giro del motor-), $119.93 \mathrm{~Hz}$ (2X) y $181.67 \mathrm{~Hz}(3 \mathrm{X})$, respectivamente, los cuales se atribuyen, a la luz de la teoría de los espectros básicos, a vibraciones ocasionadas por falta de alineamiento; esto con base en las medidas tomadas en el eje $Z$, ya que en los otros ejes no se produjo mayor efecto.

Por último, se realizó una tercera prueba de datos, en la cual, además de inducir cierta desalineación en sentido radial y aflojar los pernos del motor para sujeción a la 
base, se retiró el voltaje de alimentación de una fase. Se produjo un patrón de vibración idéntico en cada uno de los ejes, el cual se muestra en la Fig. 17, siendo de mayor magnitud en el eje Y.

En el espectro obtenido para el eje Y se observa la presencia de un pico con una amplitud de $3.5 \mu$ a una frecuencia de $1.11 \mathrm{~Hz}$, atribuido posiblemente a vibraciones por holguras debidas a tornillos sueltos. De igual manera, se identifican picos con amplitudes de $0.6 \mu, 1.3 \mu$ y $0.4 \mu$ a unas frecuencias de $62.29 \mathrm{~Hz}(1 \mathrm{X})$, $120.02 \mathrm{~Hz}(2 \mathrm{X})$ y $176.12 \mathrm{~Hz}(3 \mathrm{X})$, respectivamente. Este espectro obtenido representaría un espectro de fallas conjuntas que requieren mayor grado de análisis para determinar sus causas; no obstante, se ratifica el correcto funcionamiento del sistema diseñado, mostrando que es posible obtener los espectros de vibración para el análisis de fallas, y que a su vez indica que es posible hacer un análisis temporal.

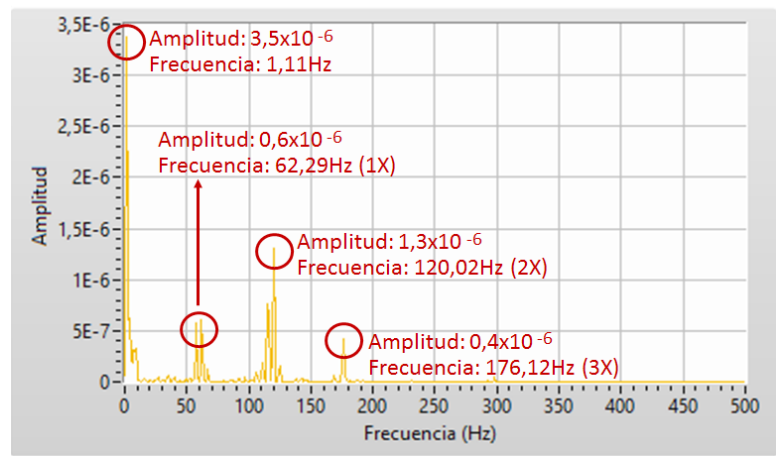

Fig. 17. Espectro de vibración de la tercera toma de datos en la prueba de funcionamiento

\section{Conclusiones}

Si bien el método de detección y análisis de fallas por vibraciones en máquinas eléctricas rotativas ha tenido grandes avances en los últimos años, y se han desarrollado equipos especiales para realizar esta labor, los cuales, debido a sus altos costos, difícilmente resultan asequibles para la formación de profesionales en instituciones universitarias, se demuestra mediante el desarrollo del sistema de análisis temporal y espectral para la detección de fallas por vibración planteado que es posible implementar un sistema como éste si se dispone de los elementos básicos y de conocimientos acerca del tema, resultando una opción muy viable para fines académicos, principalmente por factores económicos.

Con la realización del sistema de análisis temporal $\mathrm{y}$ espectral de datos para la detección de fallas por vibración en motores eléctricos se obtuvo una amplia perspectiva del poder de la herramienta que constituye el software LabVIEW, de National Instruments, tanto para la formación académica como para el campo de aplicación laboral, ya que la programación mediante diagramas de bloques realizada muestra la posibilidad de edición de las características por defecto de los bloques, de los que se dispone en el software, mostrando que puede adaptarse a las necesidades del programador, y que no se limita a un número reducido de opciones de desarrollo, abriendo aún más las posibilidades de realizar aplicaciones; además de la gran herramienta que representa a la hora de elaborar una interfaz de usuario para las aplicaciones elaboradas.

A partir de los resultados experimentales obtenidos de la realización de las pruebas de funcionamiento del sistema implementado para la detección y análisis de fallas por vibración en motores eléctricos se constata que es posible realizar el diagnóstico del estado funcional de un motor eléctrico con base principalmente en los espectros de vibración obtenidos, aunque es preciso destacar que el éxito del diagnóstico de las fallas depende en gran medida de los conocimientos y experiencia del analista en la interpretación de la información obtenida.

El sistema diseñado constituye una forma de visualizar y obtener datos característicos de señales de vibración en motores eléctricos, los cuales permiten hacer un análisis de fallas que puede ser más amplio o con mayores opciones, lo cual, precisamente, deja abierta la posibilidad de realizar un posterior trabajo para mejorar el sistema, incluyendo el reemplazo de los elementos de medición por otros de mayor fiabilidad y precisión, además de una adecuación cada vez más específica de la presentación de datos con respecto a las necesidades del analista; permitiendo y ampliando de igual modo la realización de investigación y aplicación, base fundamental de la formación de profesionales. 


\section{REFERENCIAS}

[1] F. Ramón, G. Mateo G. Diferentes tipos de vibraciones mecánicas. Universidad Autónoma de Santo Domingo, 1999. Disponible en: http:// www.monografias.com/trabajos/vibramec/ vibramec.shtml.

[2] J. Mejía M. Análisis de vibraciones en motores eléctricos asincronos trifásicos. Universidad de San Carlos de Guatemala, 2009. Disponible en: http://biblioteca.usac.edu.gt/tesis/08/08_0158_ ME.pdf.

[3] R. Torres R., C. Batista R. Análisis vibrodinámico de motores eléctricos. Universidad de Holguín, Cuba. 2010. Disponible en: http://scielo.sld.cu/scielo.php?script=sci_ arttext\&pid=S1815-59442010000100002.

[4] M. Fernández C., M. García M., G. Alfonso O., J. Cano R., J. Solares S. Técnicas para el mantenimiento y diagnóstico de máquinas eléctricas rotativas. España. 1998.

[5] D. Acosta A., J. Molina J. Adquisición de vibraciones mecánicas de un motor en funcionamiento usando Labview. Escuela Superior Politécnica del Litoral de Guayaquil. 2011. Disponible en: http://www.dspace.espol. edu.ec/handle/123456789/19459

[6] P. Saavedra, La medición y análisis de las vibraciones como técnica de inspección de equiposycomponentes, aplicaciones, normativas $y$ certificación. Universidad de Concepción, Facultad de Ingeniería, Concepción, Chile. 2011. Disponible en:http://www.aaende.org.ar/ sitio/material/CONFCHILE.pdf

[7] M. Arenas M. Diseño e implementación de un sistema de adquisición de aceleraciones con procesamiento mediante microcontrolador. Universidad de Sevilla, 2008. Disponible en: http://bibing.us.es/proyectos/abreproy/11638. 